\title{
High Accuracy Visual Servoing for Aerial Manipulation using a 7 Degrees of Freedom Industrial Manipulator
}

\author{
Maximilian Laiacker, Felix Huber and Konstantin Kondak
}

\begin{abstract}
This paper is devoted to the performance optimization of an aerial manipulation system composed of a Flettner-helicopter and 7 DoF manipulator. With experiments we demonstrate that the time delays in signal propagation between perception and actuation modules play an important role for the overall performance of an aerial manipulator system using visual servoing. We present an approach for estimation of the perception-action time delay and its active compensation based on the predicted motion of the manipulator end-effector.

Experiments show that compensating these delays improve the manipulation performance even more than elaborated methods for cooperative arm-helicopter control. The proposed approach should not be considered as a replacement of armhelicopter coordinated control but as an extension.

Additionally the reliability of the visual servoing is improved by implementing a multi object localization that is robust to occlusions of the target object.

The accuracy and robustness of the proposed visual servoing and active compensation algorithms are demonstrated in inand outdoor experiments With the proposed algorithm the aerial manipulator is able to repeatedly grasp an object with an accuracy better than $2 \mathrm{~cm}$.
\end{abstract}

\section{INTRODUCTION}

Remotely controlled manipulators have been in use for decades in underwater applications where it is dangerous or impossible to send a human to do the work. Aerial manipulation systems can be used to do this dangerous, difficult or expensive work in locations that can only be reached while flying. Examples are maintenance and inspection of tall buildings, power lines, chemical plants, bridges or work in mountain areas. Another example is manipulation and in-situ measurements during nuclear, chemical or biological disasters and accidents. In these scenarios the aerial manipulator can carry a tool, sensor or other device to the remote site where it is used in an automated fashion or controlled by an operator. The perception and control system has to perform the task of positioning the tool as precisely as possible with respect to the target object.

Many systems using multirotor platforms equipped with small lightweight manipulators have been developed in the last years. Successful aerial manipulation and building of complex structures have been presented in [1], [2] and [3] The experiments in all of these where done indoor and in a controlled environment and using an external positioning system. More recent work also include systems and experiments with aerial manipulators able to fly outdoors and use on board sensors for navigation and object localization. The

Institute of Robotics and Mechatronics, German Aerospace Center (DLR), 82230 Wessling, Germany \{maximilian.laiacker, f.huber, konstantin.kondak\} edlr.de

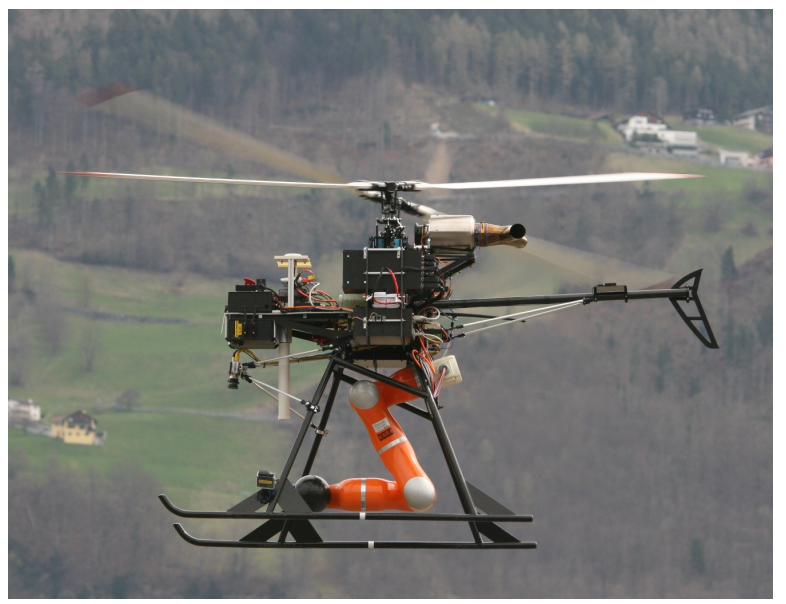

Fig. 1. Unmanned Flettner helicopter with KUKA LWR

control of an outdoor aerial manipulator using an $7 \mathrm{DoF}$ manipulator and on-board visual perception has been studied in [4]. A visual servoing control for aerial manipulation using on board cameras and marker detection to grasp a bar achieving $1 \mathrm{~cm}$ accuracy is presented in [5]. The accuracy of a parallel manipulator mounted to a quadrocopter using visual servoing to position the end-effector is evaluated in [6]. The RMS position error was around $1 \mathrm{~cm}$ and increased to about $2 \mathrm{~cm}$ when wind disturbance was added during the indoor experiments.

All the aerial manipulators mentioned above are relatively small. The system we use as shown in Figure 1 has a distance $>1 \mathrm{~m}$ between the end-effector and center of gravity. At this scale the flying platform dynamic has a great impact on the accuracy of the manipulator end-effector or tool center point (TCP). The amount of movement of the TCP when the helicopter is rotated around its axis scales with its distance from the center of gravity.

In our research work we use a system composed of an industrial manipulator with a mass of $14 \mathrm{~kg}$ which is mounted on a double rotor helicopter with a total mass of $50 \mathrm{~kg}$. The influence of the arm movement on the helicopter is significant even for slow arm movements. Therefore, attention was payed to the coordinated control of arm and helicopter as well as to the active compensation of the arm movement in the helicopter controller. As it was presented above, other research groups concentrated on the similar problems presenting approaches and methods which lead to significant improvements of manipulation performance, often in terms of TCP positioning precision. The synchronisation and signal 
propagation between perception and action modules which often run on different CPUs, was not in the focus up to now. For stationary manipulators rigidity, measurement resolution, controller performance and calibration are the dominant factors for accuracy. The dominant factor for the accuracy of a flying manipulator however is the time it takes to measure a position difference and to compensate it using the manipulator or the flying platform.

With analyzing the influence of the perception-action delay in the aerial manipulator system we designed a sensor fusion algorithm that is able to compensate the effects of time delays during aerial manipulation. Similar to other research platforms for aerial manipulation the time delays in signal propagation were minimized during the system design and are given by the used hardware and software setups. The delays themselves are not the parameters but should be considered in the control system. One possible way to do this, is presented in this paper.

The rest of the paper is organized as follows. Section II gives a brief overview of the aerial manipulator system used. The marker based target object localization is described in Section III. Section IV describes the hand-eye calibration needed for accurate visual servoing. The influence and compensation of perception-action delay is discussed in Section V. Section VI presents results from outdoor flight experiments.

\section{System OVERVIEW}

The complete aerial manipulator system as shown in Figure 1 has a takeoff mass of about $80 \mathrm{~kg}$ and can carry $10 \mathrm{~kg}$ of payload mounted to the manipulator. It is powered by a turbine engine and has a flight time of 30 minutes.

We use a Flettner helicopter configuration for the aerial platform. The Flettner helicopter has a better payload to weight ratio then the system we previously presented in [7], [8] and [9]. We use the 7 DoF KUKA lightweight robot (LWR) [10] as the manipulator for our flying system.

The helicopter autopilot uses a differential RTK GNSS receiver and an inertial measurement unit (IMU) for position and orientation measurement. The Flettner helicopter is controlled by four servos for each swashplate on the rotors. A safety pilot can take over manual control at all times if required.

The manipulator is controlled by an dedicated real-time computer. An impedance controller is used during visual servoing to be compliant when the manipulator gets in contact with the environment and reduce the interaction forces and possible damage to the manipulator [8].

The visual object localization is done by a third onboard computer that processes the images from industrial gigabit Ethernet cameras with a resolution of 1080x2048 pixel running at 30 frames per second. The cameras are mounted to the front of the helicopter looking downwards. This position was chosen because the manipulator workspace between the landing skids is inside the field of view of the cameras. All control and perception processing is done on board of the helicopter.

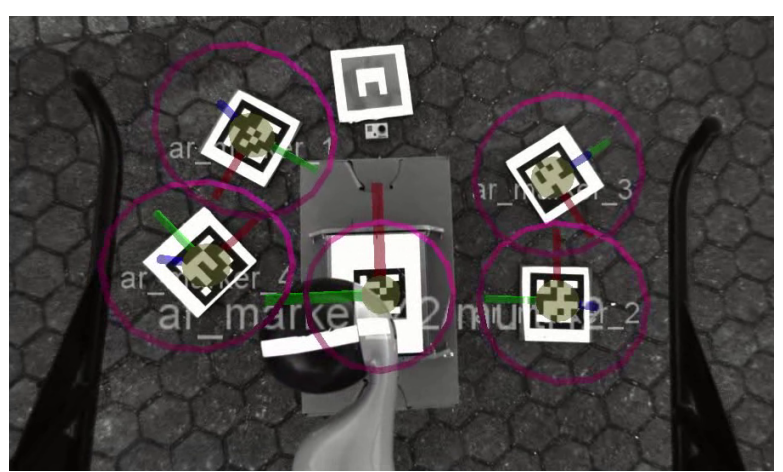

Fig. 2. Target object localized while occluded by the manipulator

\section{ROBUST MULTI MARKER OBJECT LOCALIZATION}

When manipulating an object using this manipulatorcamera arrangement the view from the on-board camera towards the object will likely be occluded as can be seen in Figure 2. This means that it is not possible to directly locate the target object during manipulation. A possible solution would be to mount cameras to the manipulator. This sensor position is problematic when the manipulator is very close to the object. The object could be out of focus or only a very small portion of the object would be in the field of view making localization difficult. Therefore we mounted the cameras to the helicopter pointing downwards with the field of view covering the workspace of the manipulator.

To compensate object occlusions we propose an algorithm that uses the localization of objects from the scene surrounding the target object. When the relative position of the surrounding objects are known this information can be used to indirectly localize the target object. We simplified the object localization task by using known artificial markers which provide in a very low false positive detection rate and accurate 6 DoF localization.

We placed multiple known markers next to the target object which is also localized using a marker. The ARToolkitPlus [11] is used for marker localization due to its fast computation when using high resolution images. The high resolution images are needed to get a high localization accuracy using a wide angle lens. The estimated accuracy of a marker with $9 \times 9 \mathrm{~cm}^{2}$ at a distance of $1 \mathrm{~m}$ with the used camera and lens setup is $1.25 \mathrm{~cm}$

The exact location of these additional markers doesn't need to be measured for the multi marker localization. The additional markers however should be near the reference marker so that they are in the field of view together with it. If the reference marker $(R M)$ mounted to the target object is localized together with another marker $\left(M_{i}\right)$, its relative pose to the reference marker $\mathbf{T}_{R M}^{M_{i}}$ can be calculated. The full 6 DoF information is needed and is stored to be used for the localization when the reference marker is occluded. If this relative measurement is done several times, the measurements are averaged to improve the accuracy by averaging out measurement noise.

If the reference marker is not detected, but other markers 
$M_{i}$ that are in the database, the position of the reference marker can be calculated using the stored pose $\mathbf{T}_{R M}^{M_{i}}$. If more than two markers are detected a RANSAC [12] algorithm is used to detect possible outliers in the marker localization. For the RANSAC algorithm only the position error between the estimated $R M$ position is used. The $\mathbf{T}_{R M}^{M_{i}}$ from the inlier together with the detected marker position in the camera frame $(C) \mathbf{T}_{M_{i}}^{C}$ are then averaged to get the estimated pose of the reference marker $\mathbf{T}_{R M}^{C}$. Figure 2 shows the localized marker positions as overlay of the camera image during a flight experiment. The reference marker is in the middle of the image partly occluded by the manipulator and localized using the multi marker localization algorithm.

\section{Automatic Hand-Eye CALibration USing MARKER LOCALIZATION}

For using the object localization described in Section III a hand-eye calibration has to be done. The transformation from the camera frame to the manipulator base frame has to be known as precise as possible to reduce the static positioning error during visual servoing. The method we use here is similar to the one described in [13] where the camera frame is calibrated relative to the GPS antenna.

For the calibration procedure a marker is rigidly connected to the tool center point $(T C P)$ of the manipulator. The manipulator is moved to different positions and orientations in a way that the marker can be detected and localized by the camera and a large portion of the field of view of the camera is covered. The detected positions and orientations of the marker $\mathbf{T}_{M}^{C}(k)$ together with the position and orientation of the TCP in the manipulator base frame $(R) \mathbf{T}_{T C P}^{R}(k)$ are recorded during the calibration motions for every time step $k$ where a marker and manipulator position is available. The manipulator position is calculated using the known forward kinematics of the KUKA LWR robot.

The homogeneous transformation parameters $\vec{p}$ for the camera frame relative to the manipulator base frame

$$
\mathbf{T}_{R}^{C}(\vec{p})=\left[\begin{array}{cccc} 
& & p_{4} \\
\boldsymbol{\operatorname { R o t }}\left(p_{1}, p_{2}, p_{3}\right) & p_{5} \\
0 & 0 & 0 & p_{6} \\
& & 1
\end{array}\right]
$$

have to be estimated. To parametrize the rotation part three Euler angles are used $\operatorname{Rot}(\phi, \theta, \psi)$.

These parameter can be found minimizing $f$ that is the distance between the marker pose $\mathbf{T}_{M}^{C}(k)$ and using the forward kinematics $\mathbf{T}_{M^{\prime}}^{C}(k)$. To define the marker pose using the manipulator forward kinematics the pose of the marker relative to the manipulator TCP $\mathbf{T}_{M}^{T C P}(\vec{p})$ is needed. This is part of the minimization problem. This pose is parameterized the same way as $\mathbf{T}_{R}^{C}(\vec{p})$. Together with the marker scale factor a total of 13 parameters have to be optimized.

$$
\mathbf{T}_{M^{\prime}}^{C}(k, \vec{p})=\mathbf{T}_{R}^{C}\left(p_{2 \ldots 7}\right) \mathbf{T}_{T C P}^{R}(k) \mathbf{T}_{M}^{T C P}\left(p_{8 \ldots 13}\right)
$$

To calculate the distance between two positions in $S E$ (3) a metric for rotation error and translation error has to be
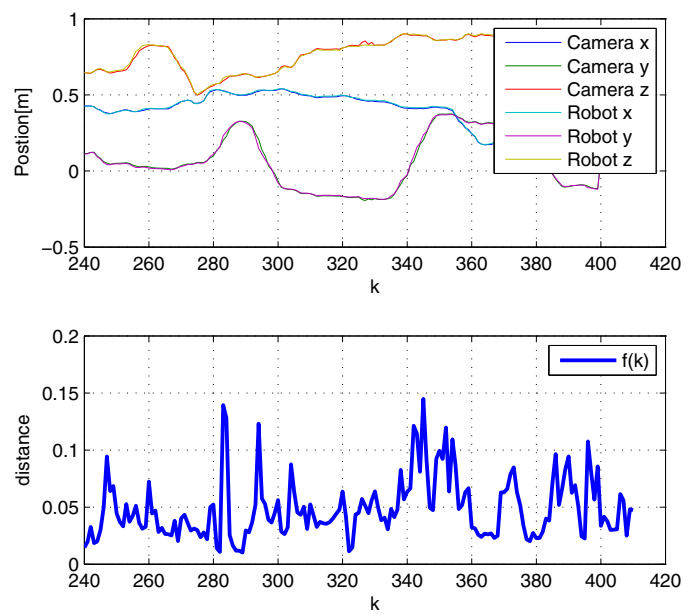

Fig. 3. Hand-Eye calibration using marker localization

defined. The Euclidean distance is used for the translation error (3). For the rotational error the magnitude of the angle in axis angle representation (4) is used as also discussed in [14].

$$
\begin{aligned}
& f\left(\mathbf{T}_{1}, \mathbf{T}_{2}\right)=g\left(\mathbf{T}_{1}^{-1} \mathbf{T}_{2}\right) \\
& g(\mathbf{T})=\sqrt{l(\mathbf{T})^{2}+(\alpha \theta(\mathbf{T}))^{2}} \\
& \mathbf{T}=\left[\begin{array}{cccc} 
& \mathbf{R} & & \vec{t} \\
0 & 0 & 0 & 1
\end{array}\right] \\
& l(\mathbf{T})=\sqrt{t_{1}^{2}+t_{2}^{2}+t_{3}^{2}} \\
& \theta(\mathbf{T})=\arccos \frac{\operatorname{trace}(\mathbf{R})-1}{2}
\end{aligned}
$$

The factor $\alpha$ can be used to weight position and orientation error. A value of $\alpha=1$ was used.

Using (2) we can define the nonlinear minimization problem for all $n$ measurements recorded during the calibration movements of the manipulator:

$$
\min _{\vec{p} \in \Re^{13}} \sum_{k=1}^{n} \frac{f\left(\mathbf{T}_{M}^{C}\left(k, p_{1}\right), \mathbf{T}_{M^{\prime}}^{C}(k, \vec{p})\right)}{n}
$$

Were $p_{1}$ is a marker scale correction factor for the marker mounted to the TCP during calibration [13]. The minimization algorithm (5) was implemented in MATLAB using constrained nonlinear minimization and run offline using the recorded data. The calculation of the minimal solution takes about a minute on a laptop with a Intel i7 M 640 using around 1000 recorded measurements. Figure 3 shows the position of the marker localized by the camera and calculated using the calibration and forward kinematics. The mean distance after calibration is 0.04 . Using the calibration method resulted in an accurate positioning of the manipulator TCP when using desired positions obtained by the optical localization. We did the calibration motions in the laboratory while the helicopter was not flying.

In future this method could also be automated and done before a flight or during a flight since the marker detection 
is also used during an aerial manipulation flight. This online calibration could be useful to compensate for modifications happening during transportation of the aerial manipulator platform making the system more robust.

\section{SENSOR DATA FUSION FOR TIME LAG COMPENSATION}

The calibration described in the Section IV minimizes the static error between the optical localization and the TCP position. For aerial manipulation the accuracy during dynamic motions is important as we have seen in different experiments. Time delays between sensor measurements and actuators are always present in real world systems and need to be addressed because they strongly influence the dynamic behavior.

The time delay between the object localization and manipulator control was measured with a target marker inside the workspace and a marker mounted to the TCP. The manipulator will start to move as soon as the target marker is detected. The marker localization will then detect when the manipulator starts to move towards the target position. This setup was used because the time delay can be measured in the same sensor context without compensating for the time it takes for the manipulator control computer to measure the manipulator position.

Analyzing the data from this experiment shows that it takes about $0.1 \mathrm{~s}$ from the moment the target marker is detected until the manipulator starts to move and even longer to reach the target position. This time lag is the perceptionaction delay and depends on how the overall system is implemented but it will never be zero. In our system the information has to pass several processing steps that add up to the perception-action delay of $0.1 s$ we observed.

When an image is captured it is transmitted over gigabit Ethernet to the vision computer where it is rectified for lens distortion. The marker is detected in the rectified image and the position is transformed to the manipulator base frame. This information is sent to the manipulator control computer that calculates the control signals for the impedance control that are sent to the manipulator actuators. Each of these steps take less than the frame interval of $\frac{1}{30} s$ but they have to be done in sequence so the processing times add up.

This delay $d$ will cause a position error $\delta x$ during visual servoing depending on the velocity of the helicopter with the well-known equation $\delta x=v d$. One could argue the helicopter is hovering during the manipulation task and $v$ close to zero which results in a very small positioning error, but actually only the helicopter center of gravity $(C o G)$ has a velocity close to zero. A point at position $\vec{r}$ relative to the $C o G$ and rigidly connected to the helicopter has a velocity of

$$
\vec{v}(\vec{r})=\vec{v}_{C o G}+\vec{\omega} \times \vec{r}
$$

with $\vec{\omega}$ being the rotational velocity of the helicopter. The point $\vec{r}$ that is important during aerial manipulation is the target object where the end-effector has to be positioned and this position should have zero velocity. With $|\vec{r}|>1 \mathrm{~m}$ in our setup and $|\vec{\omega}|>0$ most of the time this effect cannot be neglected.
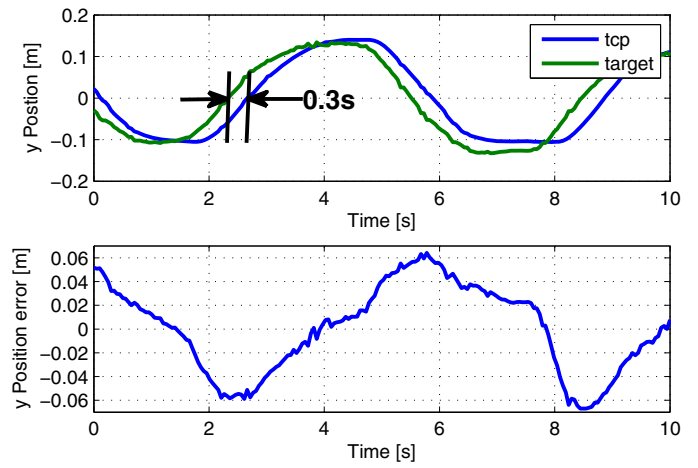

Fig. 4. Dynamic position accuracy during indoor experiment without compensating $0.3 \mathrm{~s}$ perception-action delay
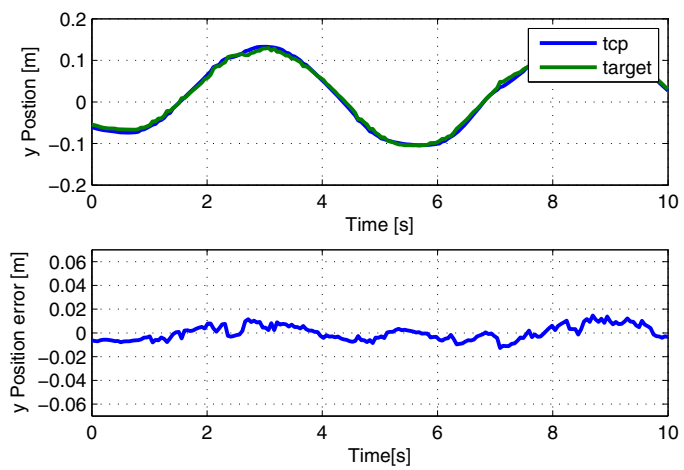

Fig. 5. Dynamic position accuracy during indoor experiment with perception-action delay compensation

In many aerial manipulation tasks the helicopter has to hover close to the ground where many external disturbances act on it. The most dominant are the ground effect and wind that create aerodynamic forces that require the helicopter to constantly adjust its roll and pitch angle to compensate position disturbances.

Our approach to compensate this time delay is to fuse the marker localization measurements with rotation velocity measurements from the on board inertial measurement unit.

From the target localization we get the homogeneous transformation of the marker in the camera frame $\mathbf{T}_{M}^{C}(t)$ at time $t$. The transformation $\mathbf{T}_{C}^{C o G}$ from the $\mathrm{CoG}$ to the camera is known. These transformations combined result in the transformation of the marker relative to the CG: $\mathbf{T}_{M}^{C o G}(t)$. We now want to know where the marker probably is at $d$ seconds in the future relative to the CoG:

$$
\tilde{\mathbf{T}}_{M}^{C o G}(t, d)=\left[\begin{array}{cc}
\mathbf{S}(\vec{\omega}(t) d) & \overrightarrow{0} \\
\overrightarrow{0}^{t} & 1
\end{array}\right] \mathbf{T}_{M}^{C o G}(t)
$$

Where $\mathbf{S}$ is the skew-symmetric cross product matrix. We neglect the translational velocity $v_{C o G}$ because it cannot directly be measured by the IMU and is close to zero during hover. This transformation is then transformed to the manipulator frame and used as the desired value for the manipulator control.

The approach was tested in an indoor experiment where 


\begin{tabular}{c|c|c} 
Look ahead time & Mean position deviation & standard deviation \\
\hline \hline $0 s$ & $0.0664 m$ & $0.0377 m$ \\
$0.1 s$ & $0.0858 m$ & $0.0436 m$ \\
$0.2 s$ & $0.0403 m$ & $0.0196 m$ \\
$0.3 s$ & $0.0339 m$ & $0.0177 m$ \\
$0.4 s$ & $0.0448 m$ & $0.0331 m$ \\
\multicolumn{2}{c}{ TABLE I }
\end{tabular}

LOOK AHEAD TIME AND POSITION ACCURACY COMPARISON

the helicopter is hanging on a crane so that the target object is in the reachable area of the manipulator. The helicopter is rotated around the vertical axis $\pm 30^{\circ}$ at different rotation velocities with and without the delay compensation. Figure 4 shows the position of the object and end-effector in helicopter $\mathrm{y}$-axis and the $\mathrm{y}$-axis position error with $d=0$. A time delay of about $0.3 \mathrm{~s}$ can be seen at the zero crossing. This delay is higher than the $0.1 s$ observed before. It includes the needed position error for the manipulator impedance controller to reach the velocity of the target object. From this we concluded we not only have to compensate the perception-action delay and tell the manipulator where the object is now but also where it probably is $0.2 s$ in the future to position the tool very close to the object when the base of the robot is moving.

Figure 5 shows the positions and distance during a similar motion when using the delay compensation with $d=0.3 s$. The position tracking accuracy was improved by a factor of 4 from a maximum of $6 \mathrm{~cm}$ to about $1.5 \mathrm{~cm}$. The impact to position accuracy during flight is discussed in Section VI.

\section{EXPERIMENTAL RESULTS}

The outdoor flight experiments were performed using the system described in Section II and shown in Figure 1. The task for the flight experiments is a pick and place operation of an object with a mass of $2 \mathrm{~kg}$.

The flight experiment starts with an automatic take-off and hover at $10 \mathrm{~m}$ altitude. After take-off the helicopter flies to the pick-up location and change the altitude to around $0.6 \mathrm{~m}$. The hover position and orientation is adjusted so that the object that will be manipulated is close to the center of the manipulator workspace. The visual servoing is enabled and the TCP is commanded to stay $10 \mathrm{~cm}$ directly above the localized object. The visual servoing error of TCP and object is constantly calculated and if this error is below a threshold for specified period of time the TCP is commanded down to grasp the object. When contact is established the helicopter will ascent to $5 \mathrm{~m}$ altitude. When the object is successfully picked-up the manipulator retracts to a park position. With the object attached to the manipulator the drop location is approached by the helicopter where the object is released. After releasing the object the helicopter performs an automatic landing at the take-off location.

During the flight experiments the performance of the multi marker localization algorithm was verified. The object was successfully localized even under difficult lighting conditions with shadows from the helicopter partly over the markers. The time delay compensation was evaluated with different

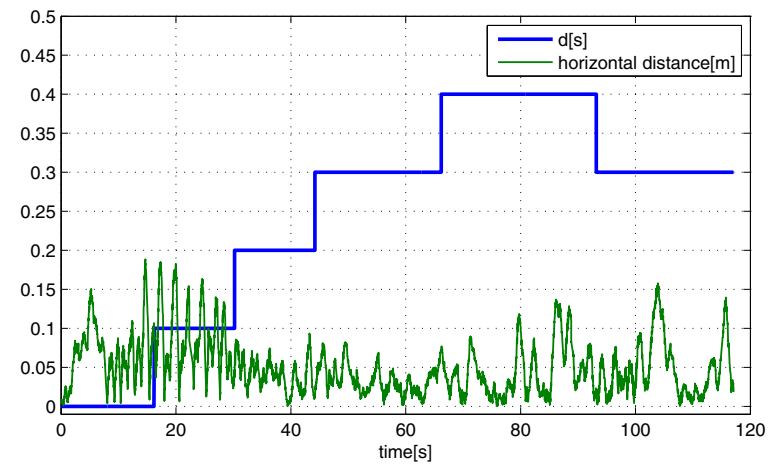

Fig. 6. Horizontal positioning deviation during visual servoing for different look ahead times

values for the look ahead factor $d$. The positioning error when the manipulator is performing the visual servoing is shown in Figure 6. The flight experiment showed that the value $d=0.3 \mathrm{~s}$ found during the indoor experiments also results the best positioning accuracy during flight. The mean and standard deviation for different settings during this experiment are shown in Table I. While finding the best look ahead factor the build up of an oscillation was observed when using $0 s$ and $0.1 s$ look ahead time. The oscillation can be seen in Figure 6 around the $20 \mathrm{~s}$ mark. The oscillation means the manipulator and autopilot are adding energy to the system which could make the whole system unstable as also discussed in [7]. With increasing look ahead time the phase of the energy flow is changed, stopping the oscillation.

Further analyzing the flight experiments shows the difference between the object localization and look ahead position to be small. A maximum difference of around $5.3 \mathrm{~cm}$ and a mean of $1.8 \mathrm{~cm}$ was recorded in the data from the flight experiments when using $d=0.3 \mathrm{~s}$. This can be explained by the more stable flight because the arm movements do not add energy to the system resulting in lower rotational velocities.

The sequence of automatically picking-up an object using the manipulator is shown in Figure 7. An electromagnet is used as the tool on the manipulator to grasp the object. Figure 8 shows the visual servoing error during a successful automatic grasping of the object. The decision when the manipulator is lowered onto the object was made automatically.

The manipulator tries to grasp the object once the horizontal distance between the TCP and the object has been smaller than a given threshold for a time period of at least $0.6 \mathrm{~s}$. At $t<0$, the threshold is $0 \mathrm{~cm}$ (grasping disabled); at $t=0$, it is set to $2 \mathrm{~cm}$; this is visualized in Figure 8. Also shown is the vertical (z) error between the object and TCP and here also the perception-action delay is visible as the time between the grasp signal and when the $\mathrm{z}$ error starts to drop at $1 s$ indicating the TCP is approaching the object vertically.

During two successive flights the manipulator was able to automatically make contact with the object. The experiments have also shown that the position error could be controlled near $1 \mathrm{~cm}$ for more than $1 \mathrm{~s}$ visible around $0 \mathrm{~s}$ in Figure 8. 


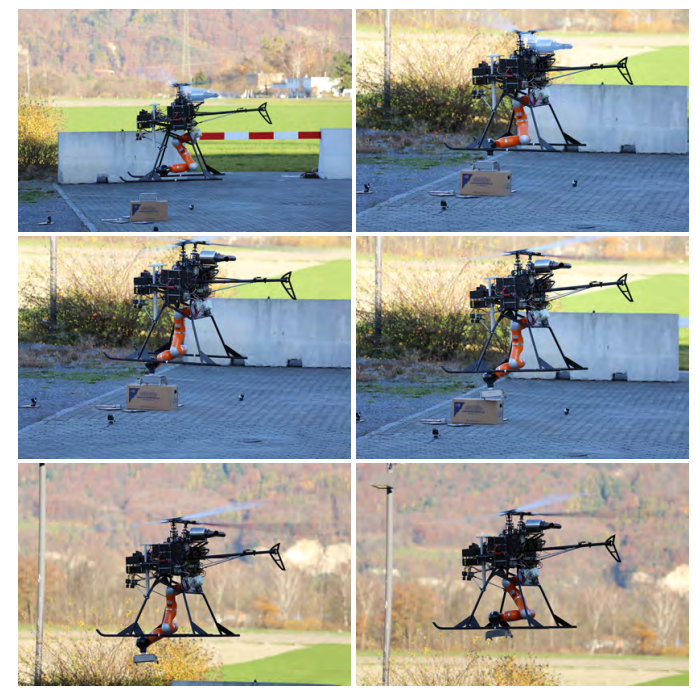

Fig. 7. Image sequence of a successfully grasping an object from the ground using the aerial manipulator

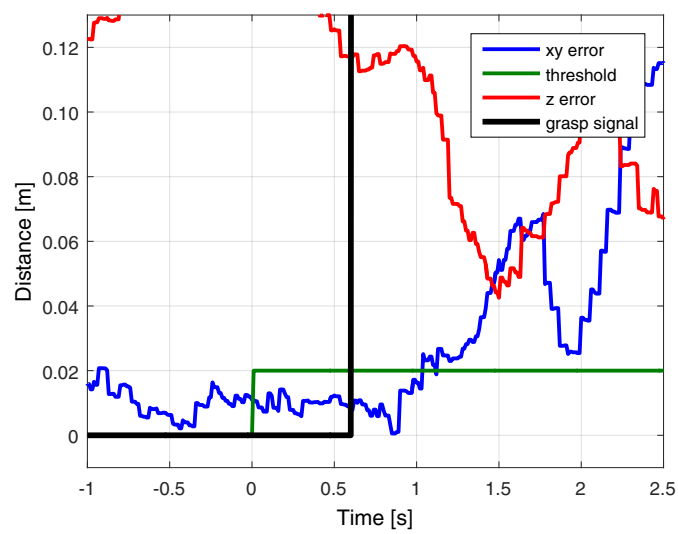

Fig. 8. Detail on position error during pick-up operation

\section{CONCLUSIONS}

In this paper we presented a robust and accurate perception system for manipulation using a floating base that is able to compensate time delays inherent in a complex system. The problem of occluding the object that is to be manipulated is solved by estimating its position relative to objects in the surrounding area. An automatic hand-eye calibration was presented that improves the static accuracy when using the visual localization to position the manipulator. To compensate the time delay that influences the dynamic position accuracy of the system a sensor fusion approach was presented. The algorithms where verified in indoor tests and outdoor flight experiments.

An automatic outdoor pick and place operation was presented and analyzed. The data showed that it is possible to position the tool mounted to the manipulator with an accuracy of better than $2 \mathrm{~cm}$ while hovering.

The ability to position the tool accurately and stable is the foundation for performing more demanding manipulation tasks like peg-in-hole insertion. Future work will also be devoted to reduce the number of artificial markers needed to localize the target object.

\section{ACKNOWLEDGMENT}

This work has been supported by the ARCAS Project, funded by the European Commission under the FP7 ICT Programme (ICT-2011-287617). We would also like to thank SwissDrones for their support during the flight experiments.

\section{REFERENCES}

[1] H. Durrant-Whyte, N. Roy, and P. Abbeel, Construction of Cubic Structures with Quadrotor Teams. MIT Press, 2012, pp. 177184. [Online]. Available: http://ieeexplore.ieee.org/xpl/articleDetails. jsp?arnumber $=6301066$

[2] D. Mellinger, M. Shomin, N. Michael, and V. Kumar, "Cooperative grasping and transport using multiple quadrotors," in Distributed Autonomous Robotic Systems, ser. Springer Tracts in Advanced Robotics, A. Martinoli, F. Mondada, N. Correll, G. Mermoud, M. Egerstedt, M. A. Hsieh, L. E. Parker, and K. StÃÿy, Eds. Springer Berlin Heidelberg, 2013, vol. 83, pp. 545-558. [Online]. Available: http://dx.doi.org/10.1007/978-3-642-32723-0_39

[3] R. Ritz and R. D'Andrea, "Carrying a flexible payload with multiple flying vehicles," in Intelligent Robots and Systems (IROS), 2013 IEEE/RSJ International Conference on, Nov 2013, pp. 3465-3471.

[4] G. Heredia, A. Jimenez-Cano, I. Sanchez, D. Llorente, V. Vega, J. Braga, J. Acosta, and A. Ollero, "Control of a multirotor outdoor aerial manipulator," in Intelligent Robots and Systems (IROS 2014), 2014 IEEE/RSJ International Conference on. IEEE, 2014, pp. 34173422.

[5] V. Lippiello, J. Cacace, A. Santamaria-Navarro, J. Andrade-Cetto, M. A. Trujillo, Y. Rodriguez Esteves, and A. Viguria, "Hybrid visual servoing with hierarchical task composition for aerial manipulation," 2015

[6] T. W. Danko, K. P. Chaney, and P. Y. Oh, "A parallel manipulator for mobile manipulating uavs," in Technologies for Practical Robot Applications (TePRA), 2015 IEEE International Conference on. IEEE, 2015, pp. 1-6.

[7] K. Kondak, F. Huber, M. Schwarzbach, M. Laiacker, D. Sommer, M. Bejar, and A. Ollero, "Aerial manipulation robot composed of an autonomous helicopter and a 7 degrees of freedom industrial manipulator," in Robotics and Automation (ICRA), 2014 IEEE International Conference on. IEEE, 2014, pp. 2107-2112.

[8] F. Huber, K. Kondak, K. Krieger, D. Sommer, M. Schwarzbach, M. Laiacker, I. Kossyk, S. Parusel, S. Haddadin, and A. Albu-Schaffer, "First analysis and experiments in aerial manipulation using fully actuated redundant robot arm," in Intelligent Robots and Systems (IROS), 2013 IEEE/RSJ International Conference on. IEEE, 2013, pp. 3452-3457.

[9] K. Kondak, K. Krieger, A. Albu-Schaeffer, M. Schwarzbach, M. Laiacker, I. Maza, A. Rodriguez-Castano, and A. Ollero, "Closed-loop behavior of an autonomous helicopter equipped with a robotic arm for aerial manipulation tasks," International Journal of Advanced Robotic Systems, vol. 10, 2013.

[10] R. Bischoff, J. Kurth, G. Schreiber, R. Koeppe, A. Albu-Schäffer A. Beyer, O. Eiberger, S. Haddadin, A. Stemmer, G. Grunwald et al., "The kuka-dlr lightweight robot arm-a new reference platform for robotics research and manufacturing," in Robotics (ISR), 201041 st international symposium on and 2010 6th German conference on robotics (ROBOTIK). VDE, 2010, pp. 1-8.

[11] D. Wagner and D. Schmalstieg, "Artoolkitplus for pose tracking on mobile devices," in Proceedings of 12th Computer Vision Winter Workshop (CVWW'07), 2007, pp. 139-146.

[12] M. A. Fischler and R. C. Bolles, "Random sample consensus: a paradigm for model fitting with applications to image analysis and automated cartography," Communications of the ACM, vol. 24, no. 6 , pp. 381-395, 1981.

[13] M. Laiacker, M. Schwarzbach, and K. Kondak, "Automatic aerial retrieval of a mobile robot using optical target tracking and localization," in Aerospace Conference, 2015 IEEE. IEEE, 2015, pp. 1-7.

[14] K. H. Strobl and G. Hirzinger, "Optimal hand-eye calibration," in Intelligent Robots and Systems, 2006 IEEE/RSJ International Conference on. IEEE, 2006, pp. 4647-4653. 